\title{
Research on Surface Roughness Prediction of Turning Parts Based on BP Artificial Neural Network
}

\author{
Ping Wang ${ }^{1, a}$, Hui Zhang ${ }^{2, b}$, Peiqing Ye ${ }^{3, \mathrm{c}}$, Tong Zhao ${ }^{4, \mathrm{~d}^{*}}$ and Qi Sun ${ }^{5, \mathrm{e}}$ \\ ${ }_{1,2,3,4}$ College of Mechanical Engineering, Tsinghua University, Haidian District, Beijing, China \\ 57 Wangjingzhonghuan Nanlu, Chaoyang District, Beijing, China \\ awang-p15@mails.tsinghua.edu.cn, ${ }^{\mathrm{b}}$ wwjj@mail.tsinghua.edu.cn \\ cyepq@tsinghua.edu.cn, dzhaotong@mail.tsinghua.edu.cn, eqi-sun@siemens.com
}

\begin{abstract}
Keywords: BP Artificial Neural Network, Surface Roughness Prediction, Levenberg-Marquardt algorithm
\end{abstract}

Abstract. Surface roughness of parts is an important index for the quality of processing. An accurate and efficient model for surface roughness prediction can provide a reliable constraint or objective function for the processing parameter optimization. In the part design and actual processing, the surface roughness value $(\mathrm{Ra})$ is taken from the national standard specification series, these series can be regarded as the corresponding category. Therefore, in this research, a new method is presented to predict the surface roughness by classifying the Ra under different cutting conditions. The effects of network structure and learning algorithm on the prediction results were discussed. Finally, a 5-3-3-3 BP Artificial Neural Network (ANN) structure with Levenberg-Marquardt (LM) algorithm were used to build the model. The results show that the prediction accuracy of the model was as high as $97.44 \%$, and the surface roughness value of the turning parts can be predicted well.

\section{Introduction}

Surface roughness is an important index to measure the quality of cutting. It is very important to establish an effective model for surface roughness prediction for the optimization of cutting process. Many scholars have done a lot of researches on the establishment of surface roughness model, which can be divided into three modeling methods ${ }^{[1]}$ : experiment- based, theoretical analysis- based and artificial intelligence (AI)-based. Experiment- based and theoretical analysis- based are generally able to achieve better predictive results, but it is difficult to apply it directly to the actual processing. The actual processing data is more uncertain because of the effect caused by the working and acquisition conditions. Therefore, the model needs to have self-learning and evolution ability, and the AI-based modeling can meet this requirement.

AI-based model have been applied by many researchers to develop model for surface roughness prediction. Ilhan Asiltürk ${ }^{[2]}$ and Mehmet Çunkas established the surface roughness prediction model of turning by ANN and multiple regression method. N.R. Abburi ${ }^{[3]}$ and U.S. Dixit predicted the surface roughness through the knowledge base. Ulas Caydas ${ }^{[4]}$ and Sami Ekici established a model of surface roughness by means of support vector machines (SVM). Azlan Mohd Zain ${ }^{[5]}$ et al. modeled the surface roughness of the milling process by ANN. Among all AI-models, the ANN is the most widely used in the fields of pattern classification and regression fitting because of its self-learning ability, adaptability, non-linearity, parallelism of computing.

The BP neural network (shown in Fig. 1) used in this paper is the most widely used neural network, which also has a lot of research on roughness prediction. Abeesh C. Basheer ${ }^{[6]}$ et al. used the LM algorithm to establish a BP ANN model, which used the tan-sigmoidal function as a transfer function with a network structure of 5-8-1. Experiments showed that the prediction accuracy and training speed of the model were much better than the traditional BP algorithm. Junde Qi ${ }^{[7]}$ and others used the LM algorithm to train the neural network model. The transfer function of the model was Tan-sigmoid and linear function. The network structure was 3-11-1, and the training abort criterion was the mean square error (MSE). D. Nathan ${ }^{[8]}$ collected the image information after cutting, and 
combined with large data mining and BP neural network to develop the model of surface roughness prediction with the network structure of 7-14-1. The results of BP neural network modeling are closely related to the number of layers, the number of hidden nodes, the learning algorithm, the performance judgment function. Therefore, this paper will discuss these aspects.

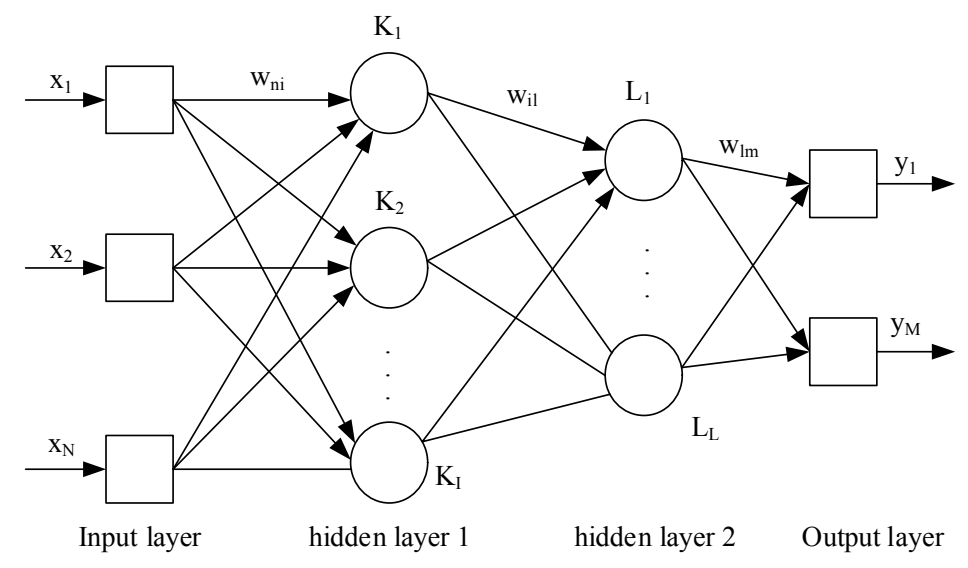

Fig. 1 BP ANN structure

In the design of the parts, the national standards have specified the surface roughness series. So in the establishment of relationship model between surface roughness and cutting parameters, it is more realistic to consider the target value as a standard series of classification values, rather than a specific surface roughness value. For this reason, this paper presents a new method to predict the surface roughness by classifying the Ra under different cutting conditions. The model of surface roughness prediction is established with full consideration of the influence of part size and cutting parameters on surface roughness. The input of the network model is the starting and ending diameter of parts, the cutting depth, the feed rate and the spindle speed, and the output is the category of the surface roughness value. The results show that the prediction accuracy of the model is as high as $97.44 \%$, and the number of iterations is 26 times, which have a very good prediction effect.

\section{Surface Roughness of Cutting Processing}

The arithmetic mean difference $(\mathrm{Ra})$ of the contours is commonly used to evaluate the surface roughness in the industry. According to GB / T 1031-2009, the value of Ra is defined in Table 1.

Table 1 Value of Ra

\begin{tabular}{|c|c|c|c|c|c|}
\hline \multirow{2}{*}{ Ra [um] } & 0.012 & 0.1 & 0.8 & 6.3 & 50 \\
& 0.025 & 0.2 & 1.6 & 12.5 & 100 \\
& 0.05 & 0.4 & 3.2 & 25 & \\
\hline
\end{tabular}

The roughness values are taken from the standard values in Table 1, both in the part design stage and the inspection stage. Therefore, the problem of roughness prediction can be transformed into the classification problem, rather than the fitting regression problem in most papers.

The surface roughness value is affected by a variety of factors, such as tools, workpieces, cutting conditions and machining parameters. The previous study mainly focuses on the effect of processing parameters on surface roughness. However, in the actual processing, when the material and size of workpiece are different, even if the processing parameters are the same, the final processing results may be different. Therefore, in this paper, the cutting parameters (spindle speed, feedrate and cutting depth) and the workpiece size (the starting and the ending diameter of the workpiece) are taken into account in the process of roughness modeling.

\section{Construction of BP Neural Network Prediction Model of Surface Roughness}

Data sources and selections. The data used in this paper is collected directly from the processing site, the processing equipment is Funuc $0 \mathrm{i} C N C$ machining center, the processing material is $40 \mathrm{Cr}$, and the 
parts are processed for the cylindrical turning. More than 400 processing examples are collected. Each sample consists of the starting and ending diameter of the parts, the spindle speed, the feed rate, the depth of the cutting and the surface roughness.

For this study, a total of 187 sample data is selected, and 148 of them as training sample data, 39 as the test sample data, the ratio of training sample data and test sample data is $3.8: 1$, this ratio is appropriate for the network training. For each sample data, select the starting diameter, ending diameter, cutting depth, feed rate and spindle speed as input data, the surface roughness category as the output data. In the sample data of this paper, the surface roughness values are 1.6, 3.2 and 6.3, so the surface roughness value is divided into categories 1,2 and 3 respectively. The sample data is shown in Table 2 (part).

Table 2 Samples data (part)

\begin{tabular}{|c|c|c|c|c|c|c|c|}
\hline $\begin{array}{c}\text { Sample } \\
\text { No. }\end{array}$ & $\begin{array}{c}\text { Starting } \\
\text { diameter } \\
{[\mathrm{mm}]}\end{array}$ & $\begin{array}{c}\text { ending } \\
\text { diameter } \\
{[\mathrm{mm}]}\end{array}$ & $\begin{array}{c}\text { Cutting } \\
\text { depth } \\
{[\mathrm{mm}]}\end{array}$ & $\begin{array}{c}\text { feed rate } \\
{[\mathrm{mm} / \mathrm{r}]}\end{array}$ & $\begin{array}{c}\text { spindle } \\
\text { speed } \\
{[\mathrm{r} / \mathrm{min}]}\end{array}$ & $\begin{array}{c}\text { Surface } \\
\text { roughness } \\
{[\mathrm{um}]}\end{array}$ & $\begin{array}{c}\text { Surface } \\
\text { roughness } \\
\text { category }\end{array}$ \\
\hline 1 & 80 & 20 & 5 & 0.28 & 700 & 6.3 & 3 \\
2 & 80.5 & 80 & 0.1 & 0.1 & 600 & 1.6 & 1 \\
3 & 11 & 10.3 & 0.7 & 0.3 & 1300 & 3.2 & 2 \\
4 & 90 & 75 & 5 & 0.28 & 500 & 6.3 & 3 \\
5 & 70.4 & 70 & 0.2 & 0.09 & 900 & 3.2 & 2 \\
6 & 45.3 & 45 & 0.2 & 0.08 & 1100 & 1.6 & 1 \\
7 & 28 & 9 & 5 & 0.22 & 1000 & 6.3 & 3 \\
8 & 16 & 15 & 0.5 & 0.08 & 1300 & 1.6 & 1 \\
9 & 25 & 10 & 4 & 0.23 & 1000 & 6.3 & 3 \\
10 & 26.4 & 26 & 0.5 & 0.08 & 1400 & 1.6 & 1 \\
\hline
\end{tabular}

Selection of network structure. The input and output layer nodes of the BP network are determined by the input and output variables. Therefore, the number of input and output layer nodes is 5 and 3 respectively. For the entire number of neural networks, the BP network may have one or more hidden layers. As the number of hidden layers increases, the speed of neural network training will slow down. In order not to make the neural network too complicated, the network will be designed with one or two hidden layers. For the number of hidden layer nodes, generally have the following reference Eq.1, Eq. $2^{[9]}$ :

$$
\begin{aligned}
& 1 \leq \mathrm{n}-1 . \\
& 1 \leq(\mathrm{m}+\mathrm{n})^{1 / 2}+\mathrm{a} .
\end{aligned}
$$

Where $\mathrm{n}$ is the number of input nodes, 1 is the number of hidden layer nodes, $\mathrm{m}$ is the number of output nodes, and a is a constant between 1 and 10. According to the formula, we can select the number of nodes as 2, 3 and 4. Therefore, we need to discuss the network structure of 5-2-3, 5-3-3, 5-4-3, 5-2-2-3, 5-3-3-3 and 4-4-4-3 as shown in Figure 2 and Figure 3. For these six network structures, we choose the best structure through the comparison of number of iterations, classification accuracy and mean square error (MSE).

Table 3 shows the training results of above six kinds of network structure, according to the table, the 5-3-3-3 type network structure has the highest classification accuracy, relatively fewer iterations and smaller number of iterations, so we choose 5-3-3-3 type network structure to establish the roughness model. 
$5 \cdot 2 \cdot 3$ structure

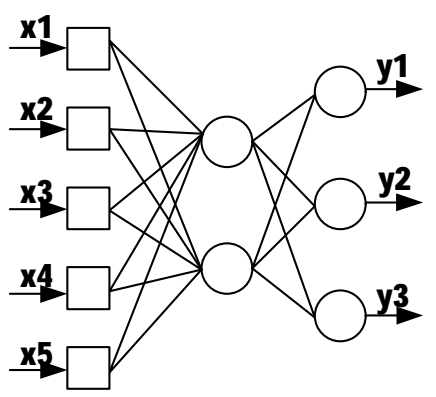

$5 \cdot 3 \cdot 3$ structure

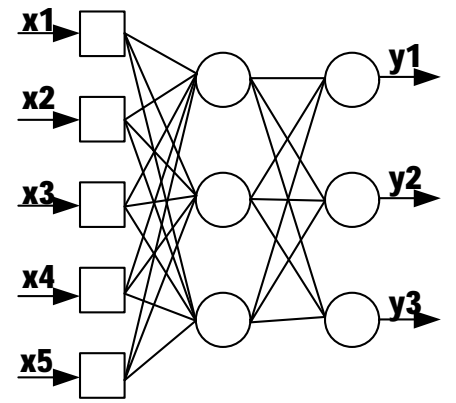

$5 \cdot 4 \cdot 3$ structure

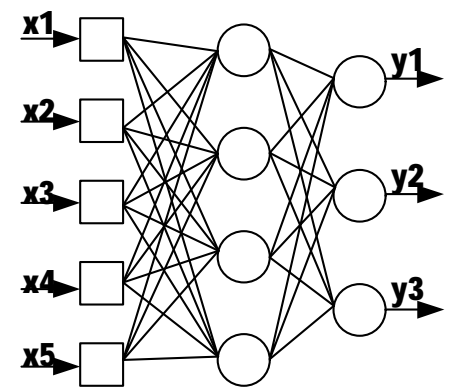

Fig. 2 Network structure with single hidden layer

$5 \cdot 2 \cdot 2 \cdot 3$ structure

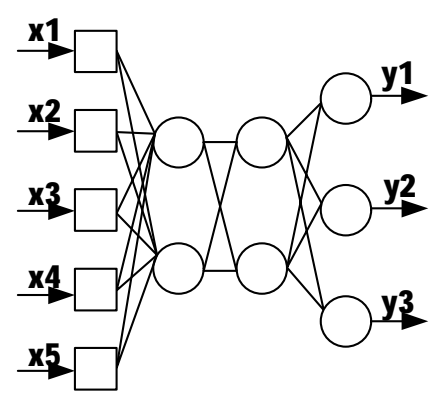

5.3.3.3 structure

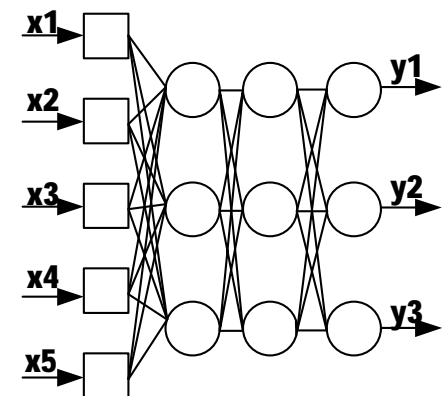

$5 \cdot 4 \cdot 4 \cdot 3$ structure

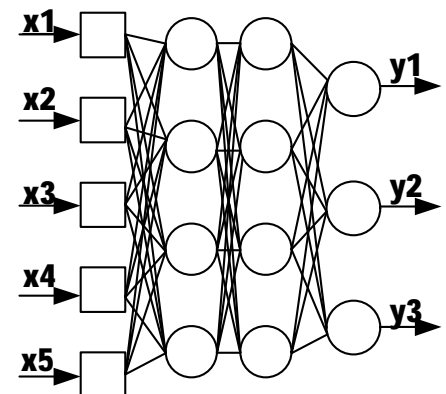

Fig. 3 Network structure with two hidden layer2

Table 3 Results of different network structures

\begin{tabular}{|c|c|c|c|}
\hline Network structure & number of iterations & MSE & classification accuracy \\
\hline $5-2-3$ structure & 56 & 0.0598 & $87.18 \%$ \\
\hline $5-3-3$ structure & 49 & 0.0349 & $92.31 \%$ \\
\hline $5-4-3$ structure & 32 & 0.0141 & $89.74 \%$ \\
\hline $5-2-2-3$ structure & 30 & 0.0247 & $84.62 \%$ \\
\hline $5-3-3-3$ structure & 72 & 0.0414 & $94.87 \%$ \\
\hline $5-4-4-3$ structure & 25 & 0.0339 & $87.18 \%$ \\
\hline
\end{tabular}

Selection of learning algorithm. After determining the number of layers of the BP network and the number of neurons per layer, it is also necessary to determine the weight factors between the layers to give the correct output value. In the training process, the weight factors change every training time. The weight factors, training speed and prediction accuracy would be different with various learning algorithms. Therefore, choosing the appropriate learning algorithm is very critical to the construction of BP neural network. Common training algorithms include gradient descent method (LMS algorithm), variable learning gradient descent algorithm (VLRBP), Levenberg-Marquardt (LM) algorithm, quasi-Newton algorithm (BFGS), elastic BP algorithm. In order to select the appropriate learning algorithm, this paper compares the training ability of these algorithms, the training results shown in Table 4.

Table 4 Results of different learning algorithm

\begin{tabular}{|c|c|c|c|}
\hline learning algorithm & number of iterations & MSE & classification accuracy \\
\hline LMS & 10000 & 0.1199 & $84.62 \%$ \\
\hline VLRBP & 120 & 0.0958 & $89.74 \%$ \\
\hline LM & 31 & 0.0273 & $97.44 \%$ \\
\hline BFGS & 30 & 0.0287 & $89.74 \%$ \\
\hline elastic BP & 72 & 0.0414 & $94.87 \%$ \\
\hline
\end{tabular}

From Table 4, it can be concluded that LM algorithm performs best with the fastest convergence speed and the highest classification accuracy, so choose LM algorithm as the learning algorithm. 


\section{Model Training and Analysis of prediction results}

In this study, we select the Machine Learning Tool Box of MATLAB 2017a as the training tool, set the relevant parameters of the training function according to the previous selection of network structure and learning algorithm. The number of training samples is 148, the number of test samples is 39, the neural network structure is 5-3-3-3, the learning algorithm is Levenberg-Marquardt, the learning rate is 0.05 , the additional momentum factor is 0.95 , the number of iterations is 1000 , MSE target value is 0.005 , validation check value is selected as 15 . When any one of the number of iterations, MSE target value and validation check value meets the conditions, the training process will stop. Figure 4 shows the training performance in the curve, Figure 5 shows the prediction effect. The results show that the training accuracy of the model is as high as $97.44 \%$, and the number of iterations is only 26 times, and it has very good prediction ability.

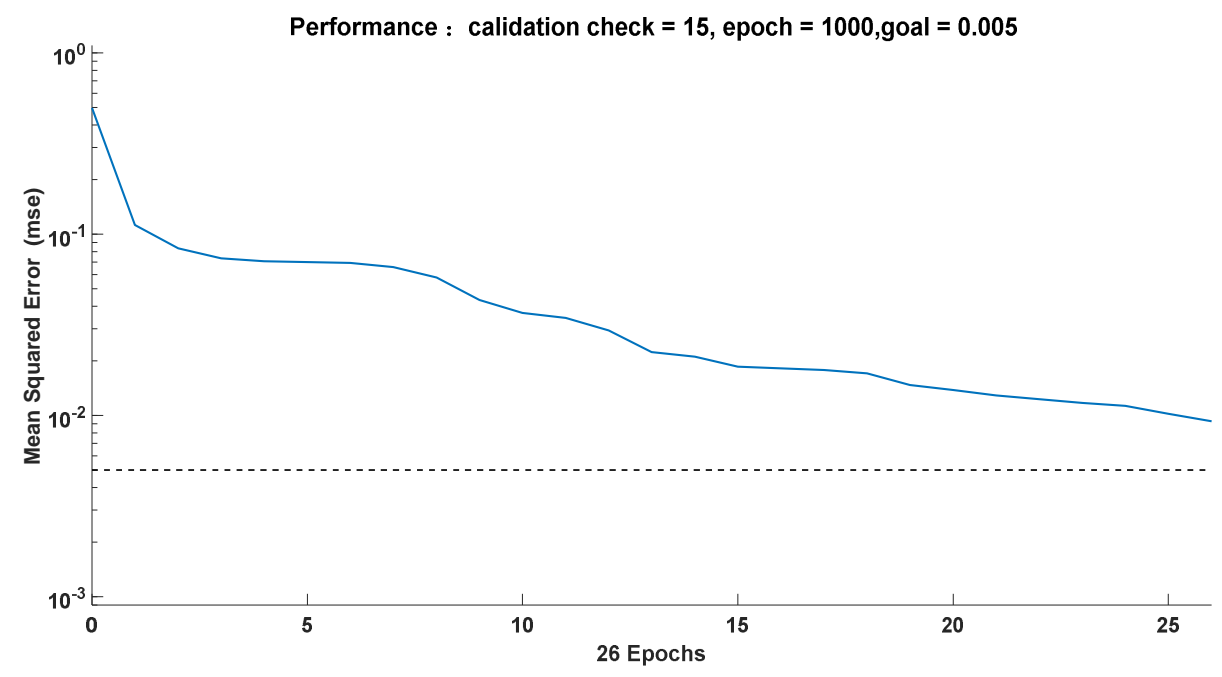

Fig. 4 Training performance

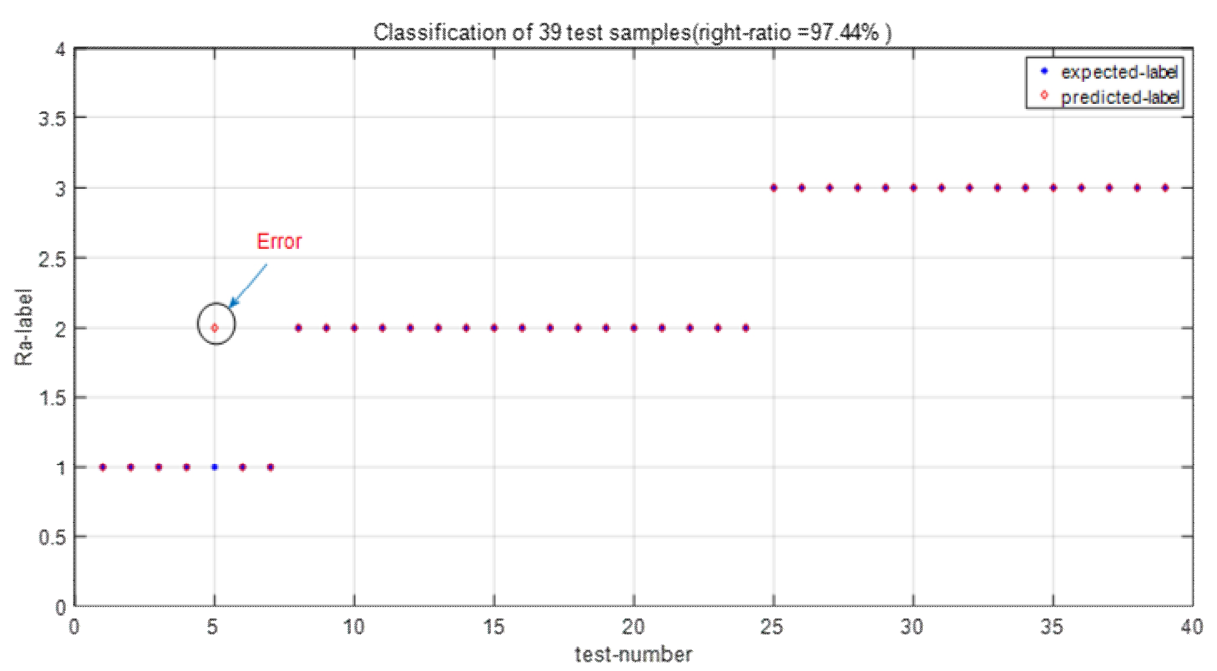

Fig. 5 Classification results

\section{Conclusions}

(1) In the part design and actual processing, the surface roughness value (Ra) is taken from the national standard specification series, these series can be regarded as the corresponding category. Therefore, this paper proposed a method to predict the surface roughness by classifying the Ra under different cutting conditions.

(2) In this paper, we summarized the influencing factors in the process of building of a BP artificial neural network. Based on the comparison of number of training iterations, MSE and prediction accuracy, the effect of different factors on model is discussed. Finally, the training network was 
chose as a 5-3-3-3 structure with the learning algorithm as LM algorithm. The results showed that the prediction accuracy of the 39 sets of test data was as high as $97.44 \%$, and the number of iterations was only 26 times, which had a very good prediction effect.

\section{Acknowledgements}

This work was financially supported by the Siemens Ltd. China.

\section{References}

[1] M. Brezocnik, M. Kovacic and M. Ficko: submitted to Journal of Materials Processing Technology (2004)

[2] Ilhan Asiltürk and Mehmet Çunkas: submitted to Expert Systems with Applications (2011)

[3] N.R. Abburi and U.S. Dixit: submitted to Robotics and Computer-Integrated Manufacturing (2006)

[4] Ulas Çaydas and Sami Ekici: submitted to Journal of Intelligent Manufacturing (2012)

[5] Azlan Mohd Zain, Habibollah Haron and Safian Sharif: submitted to Expert Systems with Applications (2010)

[6] Abeesh C. Basheer, Uday A. Dabade and Suhas S. Joshi: submitted to Journal of Materials Processing Technology (2008)

[7] Junde Qi, Dinghua Zhang, Shan Li and Bing Chen: submitted to Proceedings of the Institution of Mechanical Engineers Part B Journal of Engineering Manufacture (2016)

[8] D. Nathan, G.Thanigaiyarasu and K. Vani: submitted to International Journal of Advanced Engineering Technology (2016)

[9] Feng Shi, in: 30 Cases Analysis of MATLAB ANN(In Chinese), edited by Chinese Forum of MATLAB, chapter, 1, Beihang University Publishers(2010). 